\title{
Navigating the Root of the Mesentery: A Guided Approach to an Artery-First Pancreatoduodenectomy
}

\author{
Amer Aldouri, MD, FRCS*
}

\begin{abstract}
In the standard technique of pancreatoduodenectomy (PD), the superior mesenteric artery (SMA) is approached following Kocher maneuver of the duodenum. Alternative approaches include SMA first with no touch approaches. The aim of this article is to report the preoperative planning for and operative techniques of a combined artery first with no touch technique (CTPD) for the performance of PD. The CTPD technique is described with a detailed discussion of the operative anatomy, and of the importance of preoperative mapping using computed tomography to aid dissection of the mesenteric root and identification of the SMA. The use of careful preoperative mapping of arterial anatomy on cross-sectional imaging helps to facilitate identification of the SMA and simplifies the operative approach to PD. By incorporating detailed preoperative planning and a careful anatomical dissection, the CTPD technique provides an earlier assessment of the superior mesenteric vessels and determination of resectability.
\end{abstract}

Keywords: artery first; no touch technique; pancreatic cancer; pancreatoduodenectomy

\section{Introduction}

Despite a significant reduction in perioperative mortality, the long-term outcomes of pancreatoduodenectomy (PD) have not changed significantly over the past 30 years. ${ }^{1}$ Recent advances in the way in which pathological specimens are assessed after PD have highlighted the importance of a thorough dissection of the retroperitoneal and vascular margins in obtaining a negative (R0) resection margin. ${ }^{2}$

Dissection of the superior mesenteric artery (SMA) is a crucial step in performing $\mathrm{PD}$, and tumors found to be encasing the artery are regarded as unresectable, as evidence from the literature is not supportive of resection of the SMA in the management of pancreatic carcinoma. ${ }^{3}$ However, tumors in contact with the SMA wall for $<180^{\circ}$ of its circumference are regarded as borderline resectable and patients fitting this clinical scenario are usually referred for neoadjuvant therapy. ${ }^{3}$
Various authors have described several different techniques to identify and dissect the SMA. Sanjay et al. reviewed the literature and concluded that six techniques can be considered as artery first. ${ }^{4}$ These involved approaching the SMA from the retroperitoneum (posterior approach), the uncinate process (medial uncinate approach), the infracolic region medial to the duodenojejunal flexure (inferior infracolic or mesenteric approach), the infracolic retroperitoneum lateral to the duodenojenunal flexure (left posterior approach), the supracolic region (inferior supracolic approach), and through the lesser sac (superior approach).

The development of liver metastases despite an apparent R0 resection is a major concern after PD for pancreatic cancer, and has raised concern that manipulation of the tumor by the surgeon may potentiate this effect. This hypothesis led to the development of the nontouch technique by Kobayashi and colleagues that

Department of Surgery, King Faisal Specialist Hospital and Research Centre, Jeddah, Saudi Arabia.

*Address correspondence to: Amer Aldouri, MD, FRCS, Department of Surgery, King Faisal Specialist Hospital and Research Centre, Jeddah, Saudi Arabia, E-mail: amer2003@gmail.com

(C) Amer Aldouri 2017; Published by Mary Ann Liebert, Inc. This is an Open Access article distributed under the terms of the Creative Commons Attribution License, which permits unrestricted use, distribution, and reproduction in any medium, provided the original work is properly cited. 
has since been refined. ${ }^{5}$ Indeed a study looking for the presence of circulating tumor cells in portal venous blood during PD noted their increased presence in five of six patients undergoing conventional PD but in zero of six undergoing the no touch approach. ${ }^{6}$

We have combined the two techniques, the arteryfirst approach plus the no touch technique (CTPD). The dissection of the mesopancreas through a supracolic anterior artery-first approach can be performed without Kocher maneuver. In this article we describe the approach, which is dependent on preoperative mapping using computed tomography (CT) imaging, to help navigate the root of the mesentery and dissect the SMA.

\section{Anatomy}

The root of small bowel mesentery (RSBM) is a bare area connecting the anterior pararenal space of the retroperitoneum to the leaves of small bowel mesentery and the transverse mesocolon, with both the SMA and superior mesenteric vein (SMV) passing through the RSBM. ${ }^{7}$ Within the RSBM, the SMA is surrounded circularly by number of nerve bundles forming the superior mesenteric nerve plexus (pl-SMA). Outside the pl-SMA, there is a layer of lymph nodes embedded in adipose tissue. ${ }^{8}$ The key landmarks in the RSBM are the gastrocolic trunk of Henle (GCT) and the middle colic artery (MCA), (Fig. 1).

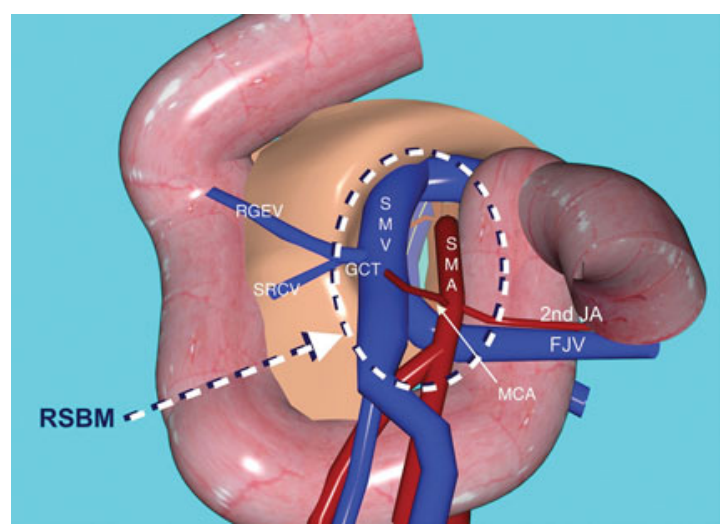

FIG. 1. Illustration of the RSBM. RGEV, right gastroepiploic vein; SRCV, superior right colic vein; GCT, gastrocolic trunk of Henle; SMV, superior mesenteric vein; SMA, superior mesenteric artery; MCA, middle colic artery; RSBM, root of small bowel mesentery; FJV, first jejunal vein; 2nd JA, second jejunal artery.

\section{Gastrocolic trunk of Henle}

The GCT represents the convergence of the transverse mesocolon, greater omentum, and the RSBM. Miyazawa et al. studied the GCT anatomy in detail and reported that this is a constant anatomical feature being identified in all 100 cases included in their study. ${ }^{9}$ In $97 \%$ of cases, both the right gastroepiploic vein (RGEV) and superior right colic vein (SRCV) drained in the GCT, in addition to small veins draining the pancreatic head. From the confluence of these veins, the GCT travels for $\sim 16 \mathrm{~mm}$ to reach the SMV, $\sim 20 \mathrm{~mm}$ inferior to the pancreatic border. ${ }^{10}$ Throughout its course, the GCT is embedded within the fatty tissue of the RSBM.

\section{Middle colic artery}

The MCA is a relatively constant branch of the SMA. It usually arises as a separate branch from the ventral wall of the SMA; however, in up to $5 \%$ of cases, it arises from the common trunk along with the other colonic arteries. ${ }^{11}$ Shatari et al. studied the relationship between the MCA and SMV in 27 cadavers and reported that the MCA ran anteriorly through the tissue to the left of SMV to reach the transverse mesocolon in all cases. $^{12}$ In another study of MCA anatomy, Horiguchi et al. studied CT imaging of 140 patients and reported that the MCA originated from the SMA distal to the inferior pancreaticduodenal arteries (IPDAs) in all cases. The exact distance between MCA and IPDA can be measured and used as a guide during the dissection. ${ }^{13}$

\section{Methods}

Preoperative mapping and its contribution

to the surgical technique of CTPD

CT imaging using a pancreas protocol is the investigation of choice before performing PD in the majority of centers, and provides a radiological roadmap of pancreatic anatomy.

The pancreas protocol is a specific triple phase imaging (noncontrast phase, late arterial phase $35 \mathrm{sec}$ after contrast administration and portal venous phase $70 \mathrm{sec}$ after contrast administration).

The first step in the mapping process is the identification of the GCT. The angle of insertion of the GCT into the SMV helps in identifying the position of the SMV (Fig. 2A). During surgery, after division of the gastrocolic ligament, the RGEV is dissected down to its confluence with the SRCV, forming the GCT. Then the GCT is followed down to its insertion into the SMV by dividing the tissue ventral to the GCT (Fig. 2B). For precise and 

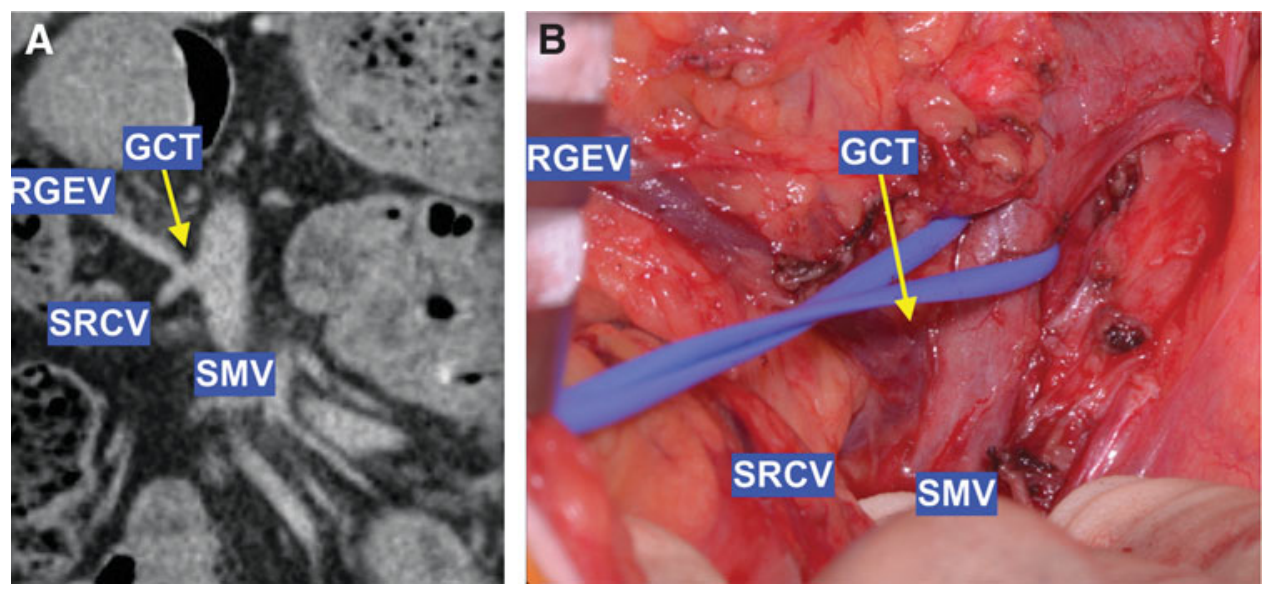

FIG. 2. The GCT dissection. (A) On coronal CT images the RGEV is easily recognized and can be followed to its confluence with the SRCV forming the GCT. (B) Operative photo shows the planned dissection of RGEV, GCT, and SMV. CT, computed tomography.

vigilant dissection at the RSBM, the author wears surgical loupes and uses the diathermy pencil with Valleylab ${ }^{\mathrm{TM}}$ Needle Electrode, 2.8" (Covidien), which curved to help the dissection around the vessels.

On CT images, the MCV drainage to SMV, or one of its tributaries, is identified in relation to the GCT. This helps to achieve an adequate surgical field exposure; if the MCV drains cranial to GCT, this may necessitate division of MCV (Fig. 3A), whereas an MCV draining caudal to GCT can be preserved (Fig. 3B).

The second step in planning the SMA dissection on axial CT images is the identification of the SMV
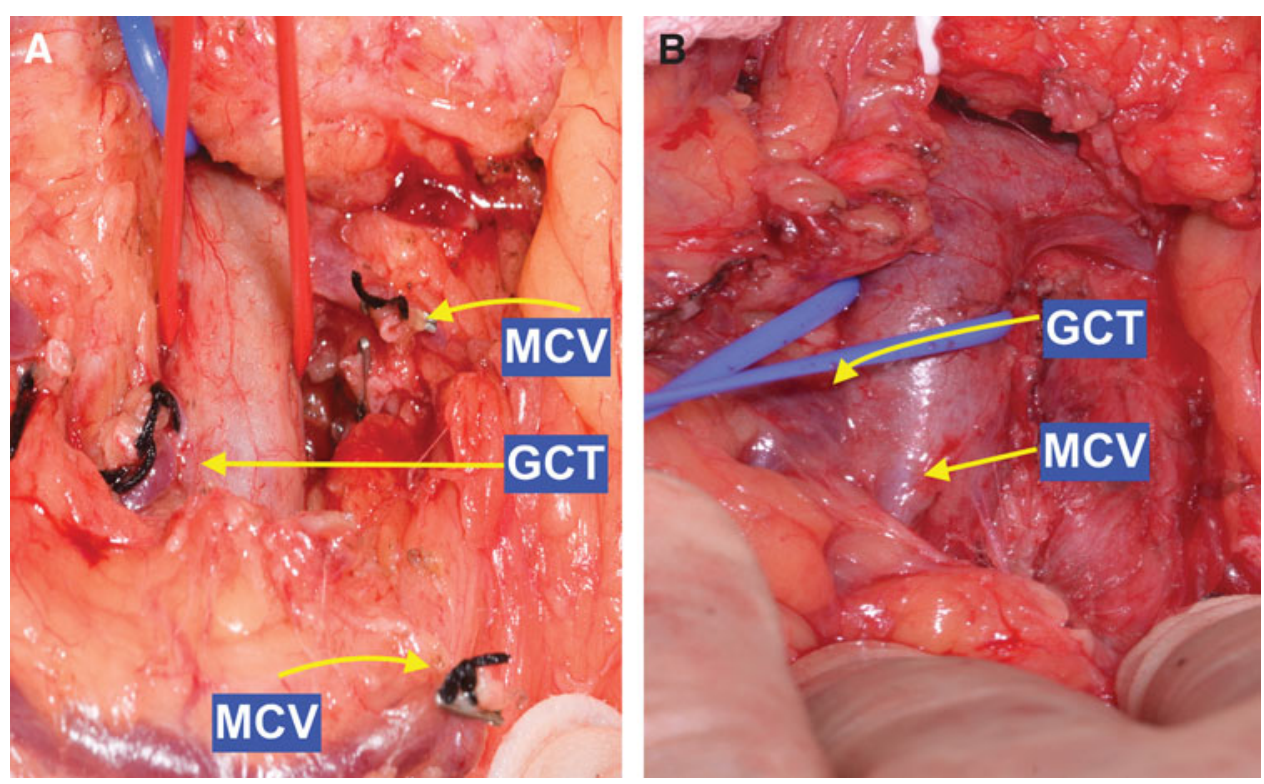

FIG. 3. Operative photograph of the MCV dissection. (A) MCV draining cranial to GCT (into IMV) the MCV was divided after ligation with silk suture. (B) MCV draining caudal to GCT (into SMV) the MCV was preserved. IMV, inferior mesenteric vein. 
groove, as shown in Figure 4A by the dotted yellow line between points $A$ and $B$. The left side of the SMV groove is related to the course of the SMA. Identification of the relationship between the SMA and SMV groove at the level of GCT insertion helps to identify the SMA.

During surgery, and after dissection of the ventral wall of the SMV, the neck of pancreas is retracted cranially to expose the insertion of the inferior mesenteric vein (IMV) into the SMV or splenic vein. The SMV is dissected off the underlying fatty tissue, exposing the SMV groove, Figure 4B.
The third step in mapping the SMA dissection on axial CT images is the identification of the distance between the SMV groove and the SMA, shown in Figure $4 \mathrm{C}$ by the yellow line between the SMA and the SMV groove. This line should extend from the ventral surface of the SMA to the nearest point on the SMV groove, to avoid the cancer infiltrated nerve bundles to the right of the SMA. The length of this line represents the thickness of the mesenteric root fat plain between the SMV groove and the SMA, and will dictate the depth of dissection to reach the SMA.
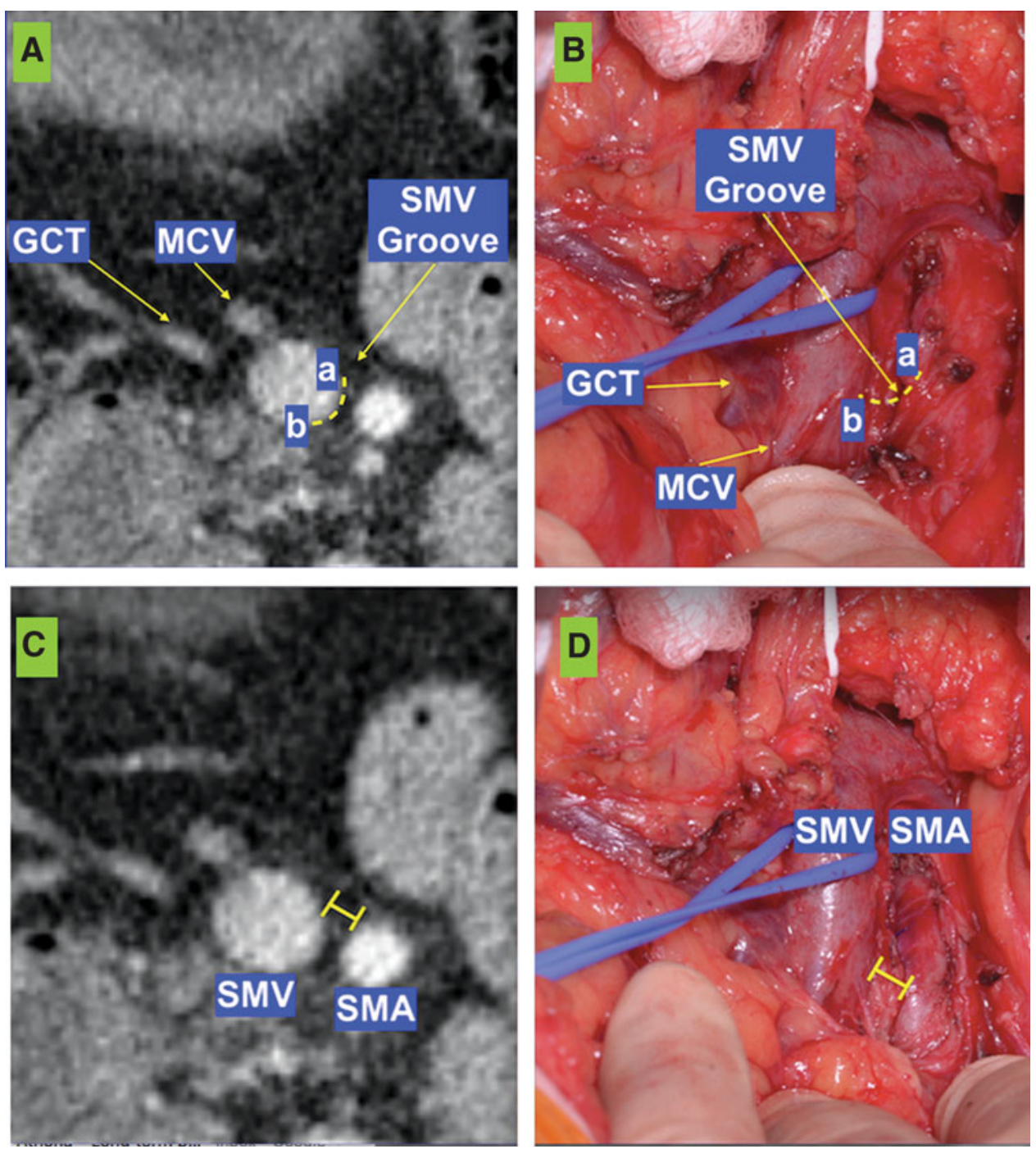

FIG. 4. CT and intraoperative images of the SMV groove. (A) The relationship between SMV groove and the SMA is identified on CT images. (B) Intraoperative photograph of SMV groove exposed after retraction of SMV to the right. (C) The thickness of SMV groove to the SMA is identified on CT images. (D) Intraoperative photograph of SMV groove division to expose the SMA ventral surface. 

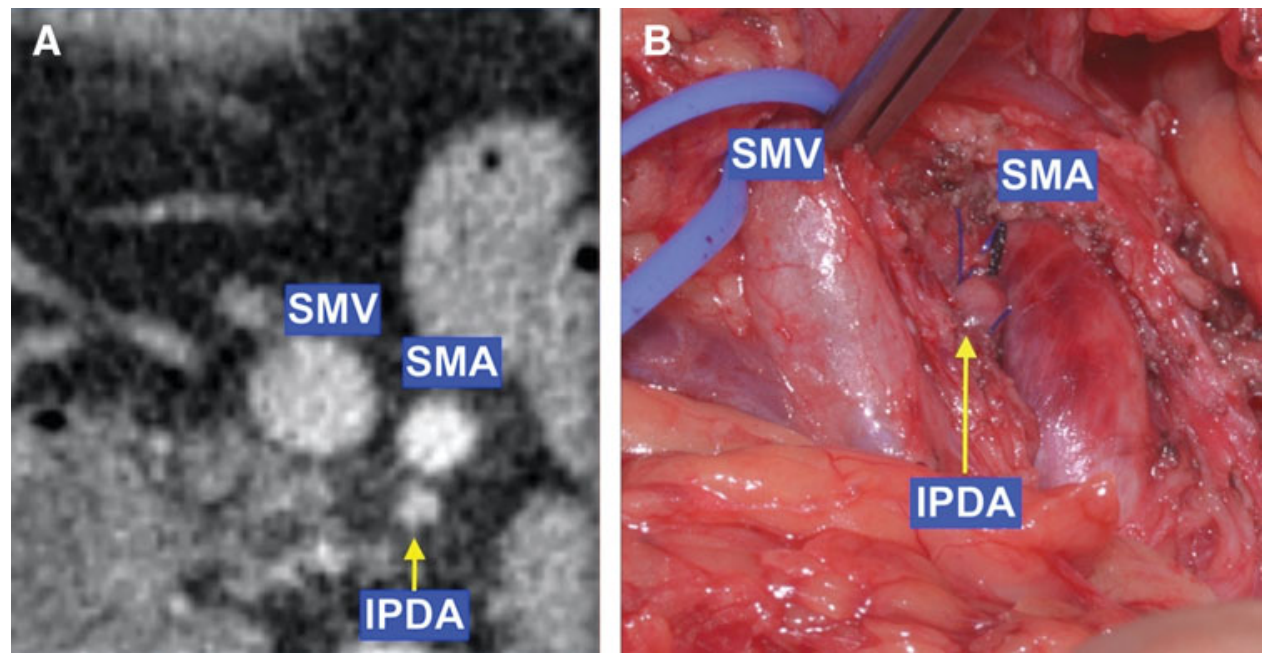

FIG. 5. CT and intraoperative images of the IPDA. (A) The IPDA is identified posterior to SMA on CT images. (B) Intraoperative photograph shows the SMA is rotated clockwise to expose the IPDA, ligated with prolene. IPDA, inferior pancreaticoduodenal artery.

During surgery, an incision is made in the SMV groove, Figure 4D. This incision extends from the identified MCA trunk at the caudal part of the SMV groove to the IMV junction with the SMV. The incision depth is increased by dividing the lymphatic-rich fatty layers between the SMV groove and the pl-SMA layer. The angle of dissection is defined by the relationship between the SMA and the SMV groove. Dividing the lymphatic-rich fatty layers will expose the pl-SMA layer that surrounds the SMA's adventitia. The right hemicircle of the pl-SMA is dissected off the SMA's adventitia. This dissection of the pl-SMA starts at the 10 o'clock position and continues toward 4 o'clock position.

Preoperative mapping using axial CT images helps to calculate the distance between the origin of the MCA and the origin of the IPDA, Figure 5A. During surgery, the SMA rotated clockwise around its longitudinal axis, exposing the origin of the IPDA on the right of the rotated SMA, Figure 5B.

The next step in the procedure is dissecting the third part of duodenum and uncinate process of mesenteric vessels, using the infracolic approach.

\section{Results and Clinical Impact}

During a period of 19 months (March 2015-August 2016), 18 patients who underwent CTPD were included in this analysis: 11 females and 7 males, with a median age of 67 years (range $37-78$ years). Histology revealed pancreatic duct adenocarcinoma in eight cases, cholangiocarcinoma in six cases, duodenal adenocarcinoma in one case, neuroendocrine tumor in one case, and main duct intraductal papillary mucinous neoplasms in two cases. The mean operative time was $481 \mathrm{~min}$ (range 333-610 min), one patient received intraoperative packed red blood cells transfusion (6\%). Mean hospital stay was 19 days, one patient was readmitted for abdominal collection (6\%). The 90 days postoperative mortality was zero. Grade A pancreatic leak was detected in two patients (12\%) and grade B pancreatic leak was detected in further two patients $(12 \%)$. One patient had reoperation, laparoscopy for bile leak $(6 \%)$.

The presence of high visceral fat (central obesity) presents a challenge to surgeons performing PD. Previous studies have demonstrated the value of sagittal abdominal diameter (SAD) both as a reliable predictor of visceral fat mass ${ }^{14}$ and as an indicator of intraabdominal operative complexity. ${ }^{15}$ The SAD of each patient was determined on axial CT by measuring the midline anterior-posterior thickness of the abdomen at the level of the fourth lumbar vertebra.

In this group of patients, the median SAD was $223 \mathrm{~mm}$ (range 178-311 mm), seven patients had significantly high visceral fat with SAD $>240 \mathrm{~mm}$; in all these cases, the artery-first approach proved to be 
feasible. However, two patients had SAD $>300 \mathrm{~mm}$, their operative times were 490 and $610 \mathrm{~min}$ and one underwent reoperation for bile leak, laparoscopic insertion of a surgical drain.

\section{Conclusions}

By incorporating detailed preoperative planning and a careful anatomical dissection, the CTPD technique provides an earlier assessment of the superior mesenteric vessels and determination of resectability.

\section{Author Disclosure Statement}

No competing financial interests exist.

\section{References}

1. Winter JM, Brennan MF, Tang LH, et al. Survival after resection of pancreatic adenocarcinoma: results from a single institution over three decades. Ann Surg Oncol 2012;19:169-175.

2. Menon KV, Gomez D, Smith AM, et al. Impact of margin status on survival following pancreatoduodenectomy for cancer: the Leeds Pathology Protocol (LEEPP). HPB (Oxford) 2009;11:18-24.

3. Bockhorn M, Uzunoglu FG, Adham M, et al. Borderline resectable pancreatic cancer: a consensus statement by the International Study Group of Pancreatic Surgery (ISGPS). Surgery 2014;155:977-988.

4. Sanjay P, Takaori K, Govil S, et al. "Artery-first" approaches to pancreatoduodenectomy. Br J Surg 2012;99:1027-1035.

5. Hirota M, Ogawa M. No-touch pancreatectomy for invasive ductal carcinoma of the pancreas. JOP 2014;15:243-249.

6. Gall TM, Jacob J, Frampton AE, et al. Reduced dissemination of circulating tumor cells with no-touch isolation surgical technique in patients with pancreatic cancer. JAMA Surg 2014;149:482-485.

7. Okino $\mathrm{Y}$, Kiyosue $\mathrm{H}$, Mori $\mathrm{H}$, et al. Root of the small-bowel mesentery: correlative anatomy and CT features of pathologic conditions. Radiographics 2001;21:1475-1490.

8. Noto M, Miwa K, Kitagawa $\mathrm{H}$, et al. Pancreas head carcinoma: frequency of invasion to soft tissue adherent to the superior mesenteric artery. Am J Surg Pathol 2005;29:1056-1061.

9. Miyazawa M, Kawai M, Hirono S, et al. Preoperative evaluation of the confluent drainage veins to the gastrocolic trunk of Henle: understanding the surgical vascular anatomy during pancreaticoduodenectomy. J Hepatobiliary Pancreat Sci 2015;22:386-391.
10. Ignjatovic D, Spasojevic M, Stimec B. Can the gastrocolic trunk of Henle serve as an anatomical landmark in laparoscopic right colectomy? A postmortem anatomical study. Am J Surg 2009;199: 249-254.

11. Gamo E, Jimenez C, Pallares E, et al. The superior mesenteric artery and the variations of the colic patterns. A new anatomical and radiological classification of the colic arteries. Surg Radiol Anat 2016;38:519-527.

12. Shatari T, Fujita $M$, Nozawa $K$, et al. Vascular anatomy for right colon lymphadenectomy. Surg Radiol Anat 2003;25:86-88.

13. Horiguchi $A$, Ishihara $S$, Ito $M$, et al. Three-dimensional models of arteries constructed using multidetector-row $C T$ images to perform pancreatoduodenectomy safely following dissection of the inferior pancreaticoduodenal artery. J Hepatobiliary Pancreat Sci 2010;17: 523-526.

14. Sampaio LR, Simões EJ, Assis AM, et al. Validity and reliability of the sagittal abdominal diameter as a predictor of visceral abdominal fat. Arq Bras Endocrinol Metabol 2007;51:980-986.

15. Clerc D, Blaser B, Demartines N, et al. Sagittal abdominal diameter is a better predictor than body mass index for duration of laparoscopic left colectomy. World J Surg 2015;39:769-775.

Cite this article as: Aldouri $A$ (2017) Navigating the root of the mesentery: a guided approach to an artery-first pancreatoduodenectomy, Journal of Pancreatic Cancer 3:1, 78-83, DOI: 10.1089/ pancan.2017.0016.

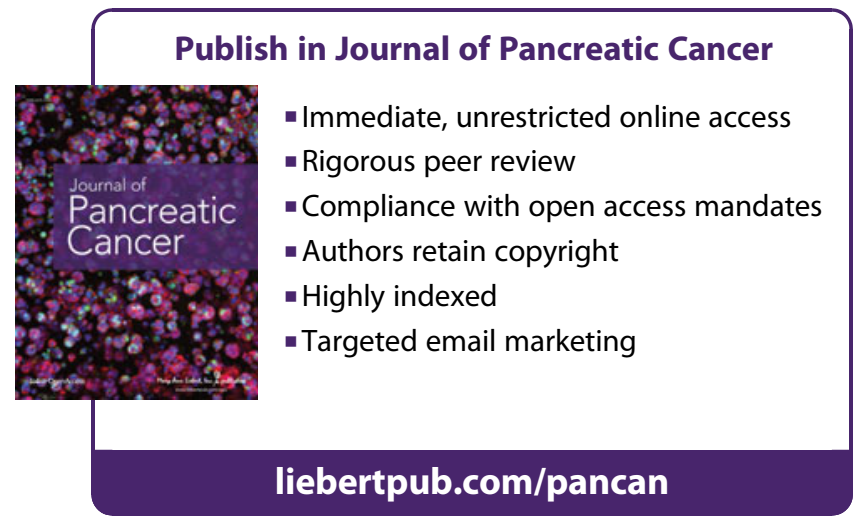

